\title{
Comparison of mean on-scene times: road versus air transportation of critically ill patients in the Western Cape of South Africa
}

\author{
D J Van Hoving, W P Smith, L A Wallis
}

Division of Emergency Medicine, Stellenbosch University/ University of Cape Town, South Africa

\section{Correspondence to:}

Dr D J van Hoving, Private Bag X25, Bellville 7535, South Africa; hoving@lantic.net

Accepted 24 September 2007

\section{ABSTRACT}

Background: The South African setting lends itself to the extensive use of air transport. There is a perception with healthcare providers that flight crews spend too much time with a patient before departure. The main advantage of aero medical transport is to minimise the delay to definitive care and prolonged on-scene time defies this objective. A study was carried out to examine the mean on-scene times of aero medical and road transport of critically ill patients in the Western Cape of South Africa. Methods: In this retrospective observational study, all critically ill patients transported in the Western Cape between September 2005 and May 2006 were evaluated. The mean on-scene time for each transport mode was calculated. Road transport was compared with air transport (rotor and fixed wing). Every transport mode was further divided into mission types: "scene" missions (scene to a healthcare facility) or "inter-facility" missions (from one healthcare facility to another).

Results: A total of 7924 transports were included in the study, 7580 of which (95.7\%) were road transports. The air transport group spent $53.2 \mathrm{~min}(95 \% \mathrm{Cl} 51.1$ to 55.4$)$ at the scene compared with $27.9 \mathrm{~min}(95 \% \mathrm{Cl} 27.5$ to 28.4) for the road transport group. There was a significant difference between scene and inter-facility missions in the air transport group (mean $31.7 \mathrm{~min}$ for scene missions vs 58.7 min for inter-facility missions; $p<0.001)$. A significant difference was also found in the road transport (mean 24.6 min for scene missions vs 31.9 min for interfacility missions; $p<0.001)$.

Conclusion: The on-scene time for transport missions by road is significantly less than for those done by air. There are significant differences between scene and inter-facility missions in both transport modes. Capacity building programmes with ongoing education and training of staff at referring facilities should be implemented.

Aero medical services have many benefits as part of an integrated Emergency Medical Service (EMS) system. ${ }^{1}$ The main advantage is to minimise the delay to definitive care, which subsequently decreases the mortality and morbidity rate. ${ }^{2}$ Air transportation is frequently used in the South African setting because of the size of the country and long distances between healthcare facilities.

There is a perception among local emergency personnel that air transportation takes too long, with the main problem being the length of time spent preparing patients before departure to definitive care. We undertook a study to test this perception. The aim of the study was to determine the mean on-scene times for patients transported by road and by air.

\section{METHODS}

\section{Setting}

The Western Cape is one of the nine provinces of South Africa. It covers $129386 \mathrm{~km}^{2}$ and has a population of approximately 4.5 million, of which 2.9 million live within the Cape Town metropole. ${ }^{3}$ There are two tertiary level hospitals in the province, both situated within the Cape Town metropole.

The South African Red Cross Air Mercy Service (AMS) has been providing aero medical services in Southern Africa since 1966, predominantly in the Western Cape. AMS is a non-profit organisation operating as an independent trust and has subsequently expanded its service to other areas of South Africa. The provincial EMS in the Western Cape embarked on an extensive aero medical programme in conjunction with the AMS which aims to complement the existing road ambulance network.

AMS has two bases in the Western Cape (fig 1). The main base is situated in Cape Town and consists of a helicopter (B3 Squirrel) and a fixed wing aircraft (Pilates PC12). The second base is situated in Oudtshoorn, $425 \mathrm{~km}$ east of Cape Town, providing service with a helicopter (B3 Squirrel). The flight crew always consists of at least one Advanced Life Support (ALS) paramedic; the second crew member is at least trained to Intermediate Life Support level. Doctors at least 2 years out of medical school can also volunteer their services as a second crew member.

The provincial EMS service has a fleet of 206 ground ambulances and has 88 operational ALS personnel (32 of whom cover the Cape Town metropole).

The Communication Centre of the provincial EMS does not use a formal triage system; all assessments are made from initial information received from the caller. Patients are triaged for dispatch into three categories: Red, Yellow and Green. The Red category is broadly defined as stretcher cases with abnormal vital signs which are deemed life-threatening. All dispatches of ground and air ambulances are done through this centre, but an air ambulance will only be dispatched after consultation with one of the four EMS doctors.

\section{Study design}

We retrospectively evaluated all critically ill patients who were transported within the Western Cape over the 9-month period from 1 September 2005 to 31 May 2006. Cases were included if they were critically ill patients (those 


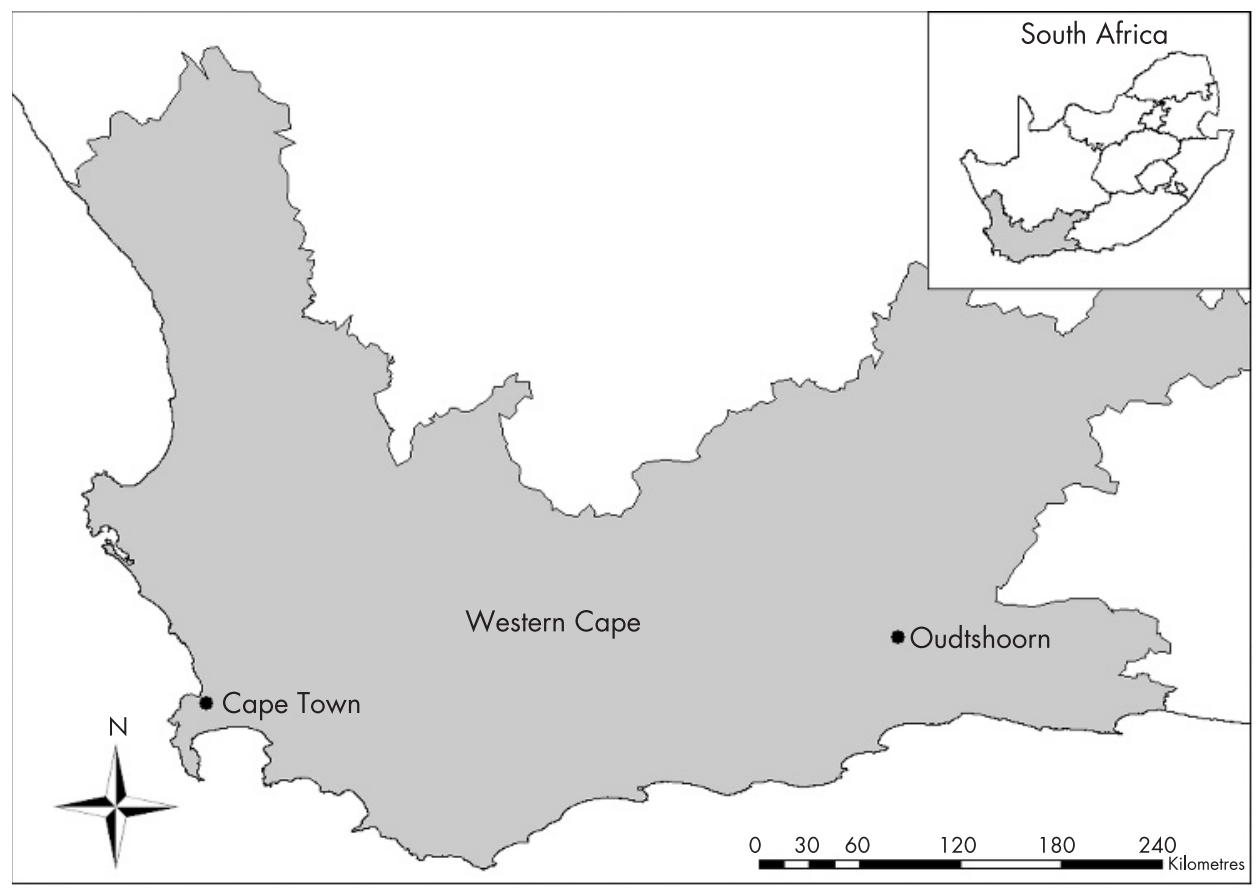

Figure 1 The Western Cape.

triaged for dispatch as Red). All cases were extracted from existing databases. The database for road transport includes all transports done by Western Cape EMS. The air transport database consists of all flights done by AMS and includes both rotor and fixed wing aircraft.

Cases were excluded from the study if:

- Transport times were not clearly documented.

- Missions were done where no patient was transported.

- Missions were done where the triage category was not indicated on the transport sheet.

- The mission type (scene or inter-facility) was not clearly indicated.

- The flight was a Search and Rescue mission.

- The flight was outgoing (departing with a patient from Cape Town to elsewhere).

All Red cases were extracted from each database. The onscene time for each transport was calculated by subtracting the arrival time from the departure time, irrespective of whether the patient was transported from an accident scene or a healthcare facility.

Every transport mode was further divided into mission types: either "scene" or "inter-facility". "Scene" missions were defined as transport from an accident scene to a healthcare facility, and "inter-facility" missions included transport from one healthcare facility to another.

\section{Analysis of data}

Statistical analyses were done by the Centre for Statistical Consultation at Stellenbosch University, using Statistica Version 7 (StatSoft Inc, 2004). Mean on-scene times of the different transport modes were compared with analysis of variance (ANOVA). A non-parametric ANOVA (MannWhitney $U$ test) was used where the residuals of the ANOVA were not normally distributed. A significance level of $5 \%$ was used.

\section{RESULTS}

A total of 7924 transports were included in the study: 7580 by road and 344 by air (fig 2). We excluded 127372 (72\%) of the total transports (triage category other than Red or not indicated); a further 41500 (84\%) were excluded: 27 air transports (19 no on-scene time indicated; 5 search and rescue; 3 outgoing flights) and 41473 road transports (30 013 no onscene time indicated; 6315 no patient transported; 5145 uncertain mission type).

There was a significant difference in mean on-scene time between the two transport modes when using one-way ANOVA: the air transport group spent $53.2 \mathrm{~min}(95 \%$ confidence interval (CI) 51.1 to 55.4 ) at the scene compared with $27.9 \mathrm{~min}$ ( $95 \%$ CI 27.5 to 28.4 ) spent by the road transport group (fig 3).

Each transport mode was subdivided into mission types (scene and inter-facility). Using two-way ANOVA, a significant interaction was detected between the transport mode and mission type $(F(1,7920)=54.3$ with $p<0.001)$ which needed further interpretation. A Bonferroni multiple comparisons procedure of the interaction effects revealed a significant difference $(p<0.001)$ between scene and inter-facility missions for air transport. A smaller though significant difference $(p<0.001)$ was also found in the road transport group. The residuals from this ANOVA were not normally distributed so the analysis was repeated non-parametrically for each transport mode. A Mann-Whitney $U$ test between missions was done for air $(p<0.001)$ and for road $(p<0.001)$ which confirmed the results of the parametric analysis.

This means that a large difference was found in the air transport group between scene and inter-facility missions (mean $31.7 \mathrm{~min}$ (95\% CI 27.1 to 36.3 ) vs $58.7 \mathrm{~min}$ (95\% CI 56.4 to 61.1$)$ ). A smaller (but still significant) difference was shown between scene and inter-facility missions in the road transport group (mean $24.6 \mathrm{~min}$ (95\% CI 24.0 to 25.2 ) vs $31.9 \min (95 \%$ CI 31.2 to 32.6$)$; fig 4 ). 


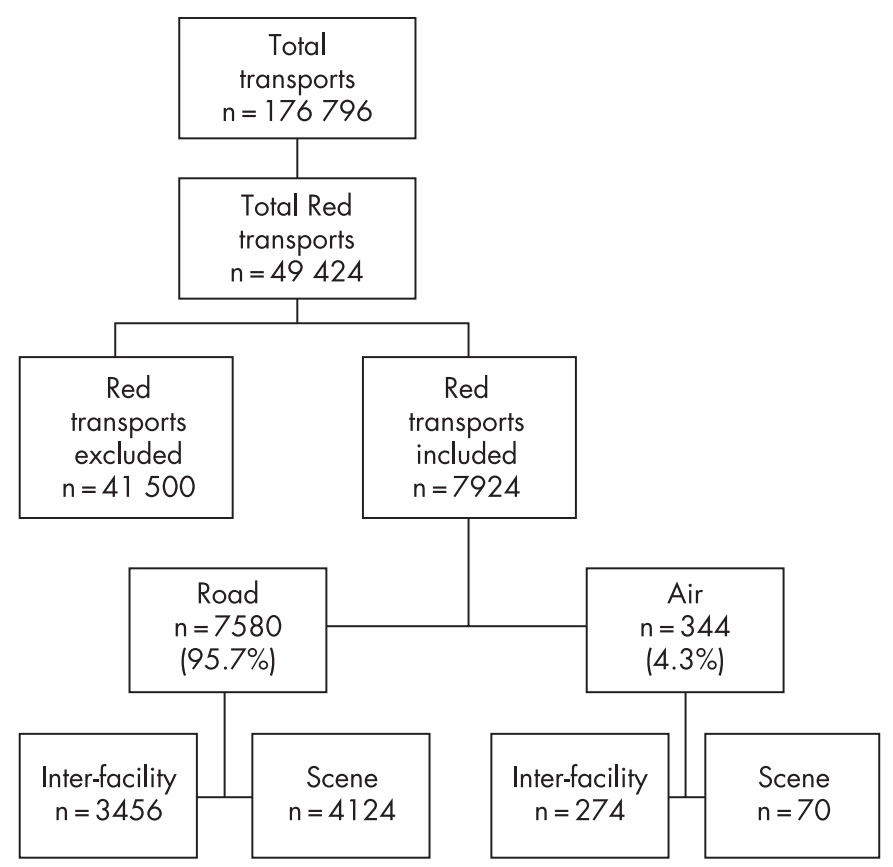

Figure 2 Total transports divided into transport mode and subdivided into mission type.

\section{DISCUSSION}

This study confirms that the air transport group spend much more time with patients before departure, almost double that of the road transport group. The main advantage of aero medical transport (to minimise time to definitive care) is thus compromised. Not only could this affect morbidity and mortality rates, but transport personnel are occupied for longer periods of time on a single mission. Efforts to reduce the onscene time interval will also reduce the total mission time and, in effect, make crews more readily available to embark on more missions. In the South African setting where all resources are limited, this is a critical issue.

A number of studies have focused on the reasons for prolonged on-scene times with air transport. It appears that it is caused by the fact that ground crews do not spend long enough, as omitted procedures by ground crews seem to be the leading cause. ${ }^{5-7}$ It has been shown that crews take longer onscene with medical patients, and even longer with children. ${ }^{89}$

The mean on-scene time for inter-facility missions in the air transport group ( $58.7 \mathrm{~min}$ ) is a concern but is in line with international findings. There is huge variation for inter-facility transport, and it seems that no established benchmark exists regarding time spent at referring facilities. Svenson and coworkers ${ }^{10}$ also showed that time spent at the referral hospital was longer for helicopter transport than for ground transport (31 (11) $\min$ vs 25 (13) $\min ; \mathrm{p}=0.008$ ).

Much of the explanation may lie in the local distribution of health care; tertiary facilities are only available within the Cape Town metropole and most of the inter-facility air transports are from places outside the metropole. These units are mostly understaffed, with junior doctors providing the clinical service, have a high turnover of nurses and have poor equipment. Previous studies have shown a direct relationship between the number of procedures omitted by emergency department personnel and the time spent at the referring facility. ${ }^{6}{ }^{11}$ Inadequate stabilisation and preparation of patients before arrival of flight crew could be due to a lack of ability or

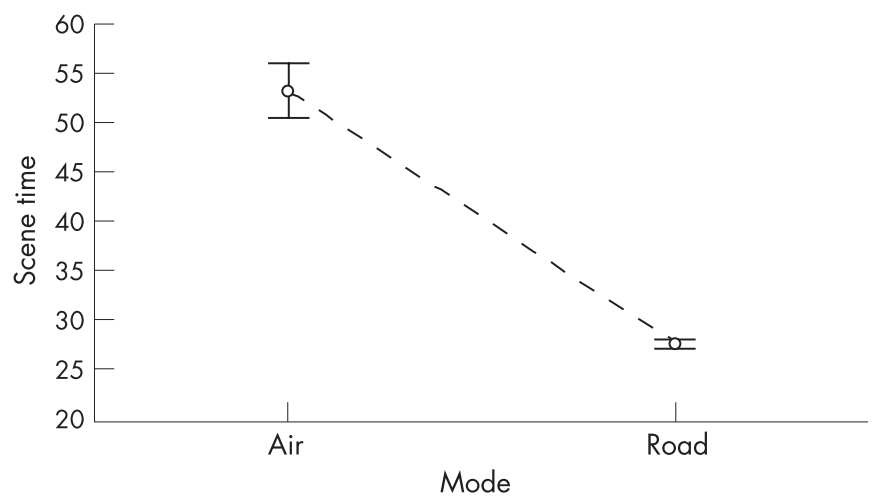

Figure 3 Plot of mean on-scene time of transport modes. Vertical bars denote 0.95 least square means confidence intervals.

equipment, or could illustrate the lack of understanding of the practical aspects and unique patient care challenges that air transport presents. ${ }^{12}$ The transport environment can be very hostile. Patients and crews are exposed to flight stressors (pressure changes, hypoxia, noise, etc) and patient management occurs with limited equipment, no support facilities and in a confined area with poor access to the patient. Pre-flight preparation is vitally important and could take up a significant amount of time.

It is perhaps not so clear cut, however. Hawkins et al ${ }^{6}$ showed that on-scene time for air transport was more than $40 \mathrm{~min}$ regardless of the number of procedures done by the crew: something other than procedures was delaying the inter-facility transport (although what this may be was not quantified). A Swedish study further indicated that the less urgent transports had the longest on-scene time. ${ }^{13}$ We did not attempt to quantify this, but it is an area that needs further research.

However, a hospital is a better environment for stabilising critically ill patients than an ambulance; it is better then to minimise the actual transport time (out-of-hospital time) without focusing on the total mission time. ${ }^{10}$ This is where the speed of aero medical transport is of greatest value. To minimise this "unstable out-of-hospital environment" seems logical. It is therefore worthwhile spending extra time stabilising a patient adequately before departing unless definitive care is only available at the receiving facility. Adequate stabilisation

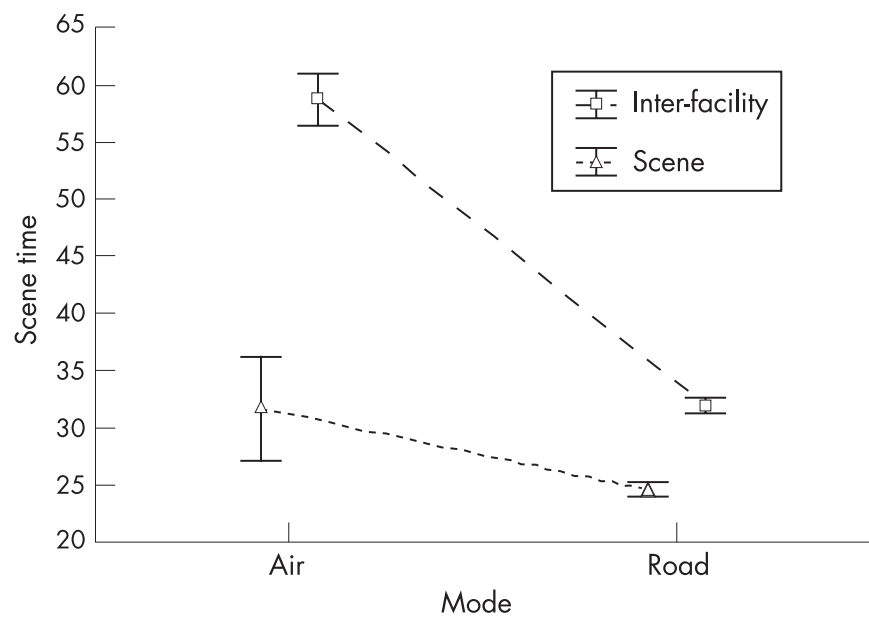

Figure 4 Plot of mean on-scene times of transport modes for scene and inter-facility missions. Vertical bars denote 0.95 least square means confidence intervals. 
of a patient prior to arrival of the transport team will help in decreasing on-scene times and needs to be emphasised, but is reliant on improvements in the emergency care system.

The on-scene time for scene missions (roadside response) also differed between the road and air groups (24.6 vs $31.7 \mathrm{~min}$ ). This is consistent with international trends. ${ }^{14}{ }^{15}$ The difference in our setting may be attributed to the shortage of ALS crews: the flight crew always consists of at least one ALS provider and therefore they spend more time on the scene performing procedures that could not be done by the non-ALS ground crew. It is also likely that the helicopter is dispatched to cases perceived as more serious by the call centre; prolonged times at the scene of complicated extrications will skew the mean onscene time upwards.

\section{Limitations}

This study has limitations. First, we analysed only 7924 of all Red transports. Only 27 of the exclusions were by air, suggesting that the data derived for the air transport group would not be significantly affected. Of the 41473 road exclusions, 6315 did not have a patient transferred so the total number of Red cases was 43109 , and we analysed $18.4 \%$ of these. The exclusion rate is indicative of the problems in local EMS services with regard to accurate patient data collection. However, we believe that the sample studied was sufficiently representative to draw meaningful conclusions.

Second, only $4.3 \%$ of transports were done by air, and this percentage drops even further if all the transports regardless of triage category are included. However, the sample size was still powerful enough for meaningful conclusions to be drawn. The use of aero medical transport in other EMS systems varies widely from $<10 \%$ to $>90 \% .{ }^{14}$ Because of restricted resources, the aero medical component of our local EMS system is underused and should be called upon more often.

Third, there was no external method available to confirm the times as entered onto the database. Errors may have occurred in transcription from the ambulance record sheets, and the data on the sheets may not have been accurate in the first place. While this may introduce an error into the magnitude of the measured differences in times, we do not believe that the overall conclusion is affected as any discrepancies would be expected to repeat across all forms of transportation.

Fourth, the study did not differentiate between case types (medical or trauma) or patient age. Paediatric and medical patients are known to take longer to prepare for transport, 89 and this may have had an impact on our results.

Finally, some of the difference in times may be explained by the fact that most of the air transports were done from rural facilities whereas most road transports were done within the Cape Town metropole. This could favour the road transport group, but the magnitude of this effect has not been quantified.

\section{CONCLUSION}

The on-scene time for transports done by road is significantly less than those done by air. There are remarkable differences between scene and inter-facility missions in both transport modes. We agree with the existing perception of local healthcare providers that flight crews spend longer with patients before transporting them to definitive care; whether they are spending too long or ground crews are not spending long enough remains to be seen. However, this perception may be leading to underutilisation of aero medical transport in the Western Cape.

We acknowledge that time at the scene is not wasted but is taken up performing essential tasks. Nonetheless, the sooner the patient gets to definitive care, the better. This must not be at the expense of adequate preparation for the journey.

Capacity building programmes need to be implemented at local referring facilities. Personnel should be trained in Advanced Life Support skills and aero medical specific courses should be implemented. Ongoing training, upgrading of facilities and equipment, and more trained personnel are needed. The importance of accurate data collection in the local EMS services should also be emphasised. The Western Cape has recently embarked on the first steps of such a programme. Future research is needed to evaluate the effects from this training programme.

Acknowledgements: The authors acknowledge the assistance of the AMS, Western Cape EMS and Professor D G Nel from the Centre for Statistical Consultation at Stellenbosch University; and salute all healthcare providers in South Africa who strive for optimal care under suboptimal conditions.

Funding: None.

Competing interests: None.

Ethics approval: Approval for this study was obtained from South African Red Cross Air Mercy Service management, Western Cape Emergency Medical Services management and from the Committee for Human Research at Stellenbosch University. Contributions: DJvH had the original idea, collected the data and wrote the first draft; all authors contributed to the final paper. DJvH is the guarantor.

\section{REFERENCES}

1. Burney RE, Fischer RP. Ground versus air transport of trauma victims: medical and logistical considerations. Ann Emerg Med 1986;15:1491-5.

2. Sampalis JS, Lavoie A, Williams Jl, et al. Impact of on-site care, prehospital time, and level of in-hospital care on survival in severely injured patients. J Trauma 1993;34:252-61.

3. Anon. Overview of the Western Cape. http://www.capegateway.gov.za/Text/2004/1/ overview western cape.pdf (accessed Apr 2007).

4. Department of Social Services and Poverty Alleviation. Western Cape population profile. http://www.capegateway.gov.za/Text/2005/7/ wc_population_profile.pdf (accessed Apr 2007).

5. Morgan S, Reidy M, Myslinski B, et al. Trauma scene analysis: identifying causes of excessive scene times. Air Med J 1995;14:170.

6. Hawkins SC, Morgan S, Waller A, et al. Effect of ground EMS and ED personnel on air medical trauma on-site times. Air Med J 2001;20:32-6.

7. Braude D, Hutton K, LaValley J. The impact of advanced airway procedures and aircraft type on rotor-wing scene times in air medical transport. Air Med $\mathrm{J}$ 2005;24:203.

8. Braude D, Hutton K, LaValley J. Benchmarking mean dispatch, lift-off and scene times in rotor-wing air medical transport scene flights. Air Med J 2005;24:206.

9. Beddingfield F, Garrison H, Manning JE, et al. Factors associated with prolongation of transport times of emergency pediatric patients requiring transfer to a tertiary centre. Pediatr Emerg Care 1996;12:416-9.

10. Svenson JE, O'Connor JE, Lindsay MB. Is air transport faster? A comparison of air versus ground transport times for interfacility transfers in a regional referral system. Air Med J 2005;24:170-2.

11. Chen $\mathbf{P}$, Macnab AJ, Sun C. Effect of transport team interventions on stabilization time in neonatal and pediatric interfacility transports. Air Med J 2005;24:244-6.

12. Martin TE. Practical aspects of aeromedical transport. Curr Anaesth Crit Care 2003;14:141-8

13. Vesterbacka J, Eriksson A. A rural ambulance helicopter system in Northern Sweden. Air Med J 2001;20:28-31.

14. Cunningham $\mathbf{P}$, Rutledge $\mathrm{R}$, Baker $\mathrm{C}$, et al. A comparison of the association of helicopter and ground ambulance transport with the outcome of injury in trauma patients transported from the scene. J Trauma 1997;43:940-6.

15. Nicholl JP, Brazier JE, Snooks HA. Effects of London helicopter emergency medical service on survival after trauma. BMJ 1995;311:217-22. 